Voix et Images

voixetimages

\title{
La condition féminine dans l'oeuvre de Gabrielle Roy
}

\section{Gabrielle Pascal}

Volume 5, numéro 1, automne 1979

Jacques Godbout

URI : https://id.erudit.org/iderudit/200193ar

DOI : https://doi.org/10.7202/200193ar

Aller au sommaire du numéro

\section{Éditeur(s)}

Les Presses de l'Université du Québec

\section{ISSN}

0318-9201 (imprimé)

1705-933X (numérique)

Découvrir la revue

\section{Citer cet article}

Pascal, G. (1979). La condition féminine dans l'oeuvre de Gabrielle Roy. Voix et Images, 5(1), 143-163. https://doi.org/10.7202/200193ar d'utilisation que vous pouvez consulter en ligne.

https://apropos.erudit.org/fr/usagers/politique-dutilisation/ 


\section{La condition féminine dans l'œuvre de Gabrielle Roy}

L'œuvre de Gabrielle Roy n'offre pas à proprement parler de discours idéologique sur la condition de la femme, mais elle présente cependant grâce à ses personnages et à plusieurs des intrigues qui la composent, certains éléments qui annoncent, le plus souvent involontairement, le discours féministe actuel. En effet, la difficulté de s'épanouir comme individu face aux exigences du rôle dans la famille, la cruauté du choix à faire entre les valeurs de l'ego et celles du groupe participent à un malaise qui, à des degrés divers, est vécu par la plupart des héroïnes. C'est en particulier dans sa peinture du rôle maternel, le plus attentivement décrit dans cette cuvre, que l'auteur développe les multiples formes d'une servitude aliénante 1 . Certains de ces personnages sont montrés comme essayant d'opposer à la fatalité de leur destin toutes les ressources de l'idéal, de la créativité et de l'imagination. Mais aucun de ces recours n'apporte de solution décisive à leur état d'asservissement et la nostalgie de leur liberté n'en paraît en général que renforcée.

La condition féminine se trouve liée à des éléments essentiels de l'esthétique de Gabrielle Roy. Le conflit vécu pas ses héroïnes entre leur rôle dans le groupe et leur soif d'autonomie individuelle recoupe par exemple, dans l'œuvre, les contradictions d'une double thématique de la mobilité et de l'attachement ou, comme le formule François Ricard, de « l'errance et de l'appartenance $2^{2}$. Prisonnières de leur rôle, les mères, en effet, se voient refuser l'alternance de l'enracinement au nomadisme qui apparait souvent comme un facteur d'équilibre dans la structure de l'œuvre comme dans la vie de plusieurs personnages. Car l'univers imaginaire de Gabrielle Roy est soumis à l'influence du mouvement ${ }^{3}$.

Contraintes de renoncer à leur liberté et en particulier à toutes les formes de l'errance dont elles partagent le goût avec les autres personnages, ces femmes fictives sont représentées comme infiniment pitoyables aux yeux de la génération qui les suit. Leur sacrifice, qui culpabilise leur entourage, marque tout particulièrement leurs filles. Chez celles-ci, ce qui apparait comme la démission maternelle provoque souvent une révolte passionnée qui compromet plus ou moins définitivement l'élaboration de leur identité féminine. Leur révolte prend la forme de sublimations diverses qui visent toutes à créer une distanciation par rapport au destin maternel. 
La représentation d'une contradiction entre la vocation maternelle, d'une part, et le goût de la liberté, "ce qui meurt en dernier lieu dans le cœur humain ${ }^{4}$, d'autre part, semble se résoudre dans une idéalisation professionnelle du personnage de l'institutrice montrée comme autonome et toutepuissante. Mère symbolique, elle répond aux exigences de l'attachement, mais personnage en transit elle garde le droit à l'errance. Nominations et déplacements rythment son existence. Son engagement auprès de ses écoliers est total, mais il est éphémère. Et sa libertè reste absolue méme quand elle la met momentanément au service d'une communauté. La maîtresse d'école apparaît en effet comme le seul personnage féminin qui domine totalement sa condition et il prend une dimension mythique dans l'œuvre. On passe ainsi de la réalité accablante du rôle maternel à une image sublimée de la vocation pédagogique. Double vision, réaliste et idéale, qui semble composer les deux faces également frustrantes d'un même destin.

Notons que de Rose-Anna, la mère accablée de Bonheur d'Occasion, à l'institutrice mythique de Ces enfants de ma vie, il n'y a pas de place dans cette œuvre pour la représentation d'un épanouissement féminin qui ne soit pas une forme, idéalisée ou non, du sacrifice. En effet, chez Gabrielle Roy, la vie de la femme est soit asservie par la maternité et son cortège d'obligations, soit exceptionnellement sublimée dans la profession d'initiatrice intellectuelle. Dans les deux cas, il y a sacrifice, d'abord à la famille, ensuite à la collectivité. Cette absence met en relief des formes qui participent a l'originalité de cette œuvre. Le réalisme des peintures de mères y dialogue, par exemple, avec le lyrisme né du pouvoir de l'institutrice toute-puissante. Au sombre tableau de mœurs répondent les promesses de l'idéal quand le mythe vient au secours du réel. Le pouvoir de la maîtresse d'école semble lié à la transmission de la connaissance par les livres et, par ce détour, la condition des femmes dans l'œuvre nous ramène à un autre thème essentiel : le propos passionné que poursuit inlassablement Gabrielle Roy sur le mystère de l'écriture. Autour du thème de la représentation littéraire du réel qui devient alors la forme ultime de la liberté, une nouvelle de Rue Deschambault fait apparaître dans l'agencement de son intrigue un dialogue très vif, nourri de contradictions.

Nous y trouvons décrite comme une aventure exceptionnelle la libération toute relative et fugitive d'Éveline, mère de famille. Et dans ce texte apparaissent la plupart des interrogations et des revendications des femmes dans l'œuvre. Les obstacles au projet de voyage de l'héroïne nourrissent en effet les développements de cette "désertion", qui devient comme une mise en abime de l'œuvre entière dans la perspective de la condition féminine.

\section{Une mise en abime: les déserteuses}

Le récit apparaît placé sous le signe des mouettes, incarnation d'une libre mobilité dont l'héroïne, Éveline, souffre d'être privée. Sa fille Christine, est la narratrice du récit et c'est à travers son regard filial que l'expérience de la mère de famille est racontée. Le spectacle du vol des oiseaux déclenche 
entre les deux femmes un dialogue sur la liberté et leur élan se transmet à Eveline pour devenir " un mouvement de son âme. "4 Par ailleurs, les mouettes reviennent périodiquement dans le texte. Réalité, elles survolent le SaintLaurent quand les voyageuses arrivent au Québec. Symbole, elles sont présentées comme des compagnes de voyage dans leurs rêves et dans leurs pensées. Et fugitivement assimilée à l'oiseau, Éveline elle-même, essayant son costume de voyage, fait tournoyer sa cape comme une aile. Elle semble alors si libre que sa fille la reconnaît à peine. Symbole de liberté, les oiseaux disparaissent pendant que le père, qui incarne l'autorité familiale, séjourne chez lui et Christine confie: «Papa regagna son poste, et les mouettes revinrent voler dans nos songes "5. Cette image subit à travers le récit une série de métamorphoses qui amènent une transformation radicale. Les oiseaux vivants qui survolaient la Rivière Rouge et faisaient rêver l'héroïne deviennent en effet, grâce à l'expérience du voyage, de palpitants souvenirs sur le visage de la conteuse. Les deux sens de l'itinéraire entrepris sont ainsi illustrés. Voyage dans le réel qui distrait Éveline de sa vie confinée et de la monotonie de l'Ouest, il est aussi et surtout un voyage qui exprime son désir d'espace. Pour sa fille Christine, qui en narre les péripéties, leur itinéraire est un modeste voyage de formation. Par ailleurs, c'est sur cette image récurrente que prend appui la structure rigoureuse de cette nouvelle et la réduction qu'elle subit, du réel au symbole, apparaît comme le résultat de l'alchimie intérieure vécue par l'héroïne au cours de sa brève émancipation. Éveline s'est en effet approprié les oiseaux qui défiaient son imagination au-dessus de la Rivière Rouge. Immortalisés dans sa mémoire, ils sont désormais partie d'elle-même: sur son visage, ses souvenirs sont comparés à « des oiseaux en plein vol $» 6$.

\section{La conquête de la liberté}

Le voyage que font ensemble Éveline et Christine naît d'un défi que la fille a lancé à la mère. Celle-ci a confié qu'elle a encore envie d'être libre, ce qui contrarie Christine qui partage avec sa génération une pitié condescendante pour sa mère. Mais contrairement au silence que l'on trouve entre mères et filles dans Bonheur d'Occasion et la Rivière sans repos, par exemple, dans le milieu petit-bourgeois de la Rue Deschambault, les filles ont des conversations avec leurs mères et leur relation conflictuelle est feutrée. Christine essaie cependant de ramener sa mère à la rèalité. En effet, qu'est-il ressorti jusqu'ici des ambitieuses conversations de celle-ci sur la liberté sinon "plus d'enfants encore, beaucoup plus de couture, beaucoup plus d'ouvrage»? Cette mise au point n'entame pas l'assurance d'Éveline qui soutient en retour que rien n'émousse dans les consciences l'acuité des désirs, qui survivent étrangers au réel quand il le faut. Elle affirme en particulier que sa «captivité " n'a pas usé en elle une disposition pour la liberté. De ce dialogue de sourds va naître une aventure partagée.

Témoin sévère des contradictions de sa mère, Christine joue un rôle ambigu, car elle est à plusieurs reprises le porte-parole du milieu et, à ce titre, elle tend à castrer les espérances de sa mère comme par exemple quand elle 
lui demande si la vie ne lui a pas tout accordé en lui donnant une maison, un mari et des enfants. Elle se sert alors de l'idéologie ambiante pour dissimuler l'insécurité que lui inspire le désir de liberté de sa mère et son inquiétude à l'idée que celle-ci puisse désirer autre chose qu'être à jamais captive des siens. A ce titre, elle incarne plus précisément les réserves du milieu familial face à l'émancipation d'Éveline. Elle confie d'ailleurs qu'elle compte sur les autres membres de la famille pour résister à Éveline. Résumant ainsi toutes les résistances au projet de sa mère, elle admet souhaiter que ceux qu'elle aime soient "heureux dans leur captivitè " 8 . Le combat qui se livre dans le cœur de Christine est un modèle du genre, car il présente successivement toutes les pressions qui s'exercent sur la mère de famille pour l'empêcher de réaliser son modeste, mais très réel projet de voyage.

Chaque étape qui rapproche Éveline de cette réalisation lui coûte un effort d'affirmation auquel elle doit consentir. Elle ressemble alors à ces héros des contes qui doivent traverser diverses épreuves pour atteindre l'objet de leur désir. A ce titre, le but d'Éveline est présenté comme un exploit à réaliser et les préparatifs de son départ comme une sorte d'initiation pour l'héroïne. Le plus difficile pour elle n'est pas de travailler la nuit, toutes ses autres tâches terminées, à son costume de voyage, ni de coudre pour sa voisine afin de gagner un peu d'argent. C'est, par exemple, de couper les cheveux de ses cadettes qu'on refuse d'accepter en pension avec leurs nattes. Il est donc montré que c'est l'idée qu'elle se fait de son rôle et de ses devoirs qu'Éveline doit modifier pour se libérer. Car, en l'occurrence, les deux fillettes sont ravies de se faire couper les cheveux et de devenir "comme des garçons libres de leur chevelure "9. On voit l'héroïne obligée de renoncer à des habitudes qui font son existence et dont les chaînes résultent parfois de ses propres choix. Obligée de défaire tant de liens, elle en devient malheureuse. La narratrice qui observe sa mère constate ainsi que "la liberté non plus ne laisse pas beaucoup de repos au cœur humain $\$ 10$.

Pourtant, en dépit de ces chaînes visibles et invisibles qui retiennent Eveline à son foyer, la volonté l'emporte sur la pusillanimité de la routine. Les deux voyageuses prennent le train et l'aventure qui commence opère une immédiate transformation sur l'héroïne. Christine croit remarquer en effet qu'elle rajeunit à vue d'œil tandis que ses yeux deviennent "tout pleins d'étincelles "11 qui jaillissent à la vue de presque tout. C'est comme si, après lui avoir longtemps été dérobée, la beauté du monde lui était rendue.

\section{Les obstacles symboliques}

Le succès de son entreprise ne va pas sans nourrir chez Éveline un obscur sentiment de culpabilité. En partant, elle demande à sa fille Odette, devenue religieuse, de prier pour un projet qu'elle n'ose pas lui confier. Le souvenir d'avoir quitté son foyer en l'absence de son mari la poursuit. C'est pourquoi elle quête les signes d'une autorité supérieure qui puisse remplacer cette autorisation manquante. Quand elle se voit commander un costume par une de ses voisines, elle se dit par exemple que si Dieu lui donne la possibilité 
de gagner ainsi de l'argent, c'est parce qu'il tient à ce qu'elle parte. En recevant par ailleurs dix dollars de son frère, elle voit là un autre signe du Ciel. Même après son départ, sa quête de l'approbation se poursuit. Lors de sa visite au frère André à Montréal, elle lui demande si ce qu'elle a fait est un péché. Puis, à Saint-Anne de Beaupré, elle se met sous la protection de la Sainte pour que Christine écrive à son père que ce pèlerinage est "le vrai but "12 de leur voyage.

Née de la culpabilité que lui inspire son geste de libération, cette anxiété trouvera une fugitive satisfaction auprès de son ancienne amie de couvent, Odile, devenue Sœur Étienne. C'est elle qui lui dira qu'il y a bien des façons d'obéir à Dieu et que "la liberté est un des chemins pour aller à lui »13. Pourtant, malgré cette assurance, on voit bientôt le malaise de la "déserteuse" reprendre et augmenter avec le temps de son absence. Dans le train du retour, elle est assaillie par des inquiétudes précises et sa fille la voit vieillir tandis qu'elle laisse les scrupules l'assaillir. Éveline imagine en effet successivement chacun de ses enfants, puis son mari, victimes d'une manière ou d'une autre de son absence. Elle trouve aussi que le train n'avance pas. Et le paysage semble participer de ce malaise en n'offrant plus que des bois brûlés et des abattis. Une de ses compagnes de voyage résume toute la désapprobation qu'elle vainc en lui disant que jamais, elle, ne s'absente du foyer conjugal. Et un rêve vient finalement résumer l'anxiété née de cette laborieuse et fugitive libération: Éveline s'y voit seule au monde et obligée de retrouver ses enfants éparpillés dans tout le Canada.

\section{L'exploit}

C'est donc comme des coupables que les voyageuses rentrent au bercail où le père de famille, revenu plus vite que prévu, les attend avec les autres enfants. Elles apprennent que depuis dix jours, il marche dans les couloirs de la maison, sans que personne n'ose lui adresser la parole. II reste donc à Éveline à traverser l'épreuve finale, celle du pardon. On voit alors son personnage subir soudain une métamorphose. Toute anxiété disparaît de son visage et de son discours pour faire place à une assurance souriante. Malgré le silence réprobateur qui l'accueille, malgré l'anathème jeté par son mari en les voyant: "Mes déserteuses!»14, elle entame avec aisance le récit de son voyage. Avec art et diplomatie, elle commence par parler à son mari de sa famille à lui où elle est allée retrouver ses souvenirs d'enfance. Elle ajoute habilement avoir voulu réparer l'oubli des années, se donnant ainsi un rôle moral qui la met en valeur. Par la magie de son discours, elle fascine son auditoire en présentant des descriptions stylisées pleines de lyrisme. Bientôt ses enfants s'approchent pour voir de plus près ses yeux qui leur semblent, avant ses paroles, annoncer les paysages. Finalement, le père, entièrement métamorphosé par le récit qu'il entend, verse une larme d'émotion et demande timidement d'autres détails sur la maison de son enfance, tandis que la voyageuse garde toute la famille dans le cercle enchanté de ses évocations. Pour cet exploit décisif, c'est le pouvoir du verbe qui permet à Éveline de reprendre le contrôle d'une situation perdue. C'est cette subli- 
mation littéraire qui donne à l'auteur la possibilité de terminer la nouvelle par une fin heureuse.

A la mère de famille décrite comme culpabilisée par son départ s'est substitué un personnage mythique, celui de la conteuse qui par la puissance de son imagination et les séductions de son talent transcende sa personne et les circonstances. Au secours d'Éveline, l'artiste vient dresser un tableau dans lequel le passé et le présent se confondent pour mieux faire absoudre "la déserteuse". Son juge désarmé redevient un enfant seulement préoccupé de voir s'éterniser ce moment privilégié. Et dans une sorte de transfiguration, Eveline est montrée avec un sourire triomphant que composent sur son visage ses récents souvenirs.

A la description réaliste des épreuves de l'heroïne et de ses inquiétudes, on voit ainsi succéder son double mythique, sorte de deus ex machina, qui réalise l'exploit de s'affirmer. Au talent réaliste de la narratrice répond une envolée lyrique dans laquelle se résolvent les problèmes nés de la représentation du réel. Le tableau de mœurs fait place au merveilleux. Nous verrons que le recours mythique, qui joue un rôle décisif dans cette nouvelle, se retrouve dans d'autres ceuvres de Gabrielle Roy. Dans Les Déserteuses, il apparaît comme une tentative de métamorphose de la situation de la femme.

Cette nouvelle résume plusieurs éléments importants de la condition féminine telle qu'elle apparaît dans l'œuvre de Gabrielle Roy. Le personnage d'Éveline incarne le dévouement des mères, mais aussi la soif de liberté des prisonnières du rôle maternel, telles que souvent décrites. Christine représente la jeune génération qui critique les mères de s'être laissé asservir et entend bien choisir une autre existence. On trouve aussi les résistances du milieu au projet de la mère de famille chez Christine et trois personnages féminins qui désapprouvent sa libération. Pour résoudre le conflit né de l'émancipation de son héroïne, l'auteur choisit de la remplacer par son double, investi de tous les pouvoirs. Ce recours au mythe comme médiation entre l'exigence de liberté et la vocation maternelle apparaît comme un élement essentiel de la condition téminine dans l'œuvre et nous la verrons trouver sa forme achevée dans l'héroïne de Ces enfants de ma vie. C'est ce personnage qui oppose sa parfaite autonomie au destin accablant des mères asservies.

\section{Les prisonnières}

De tous les rôles féminins, c'est celui de la mère qui est le plus longuement décrit dans l'œuvre. En faisant l'analyse des images de femme, on découvre en effet que la plupart sont montrées dans ce rôle, oppressées par leur fonction. Aliénées, elles apparaissent comme ayant perdu la jouissance de leur personne au profit de leur entourage.

Certains de ces personnages ne font qu'une fugitive apparition dans le texte et cet écrasement, moral ou matériel, est alors la seule réalité que la 
narratrice livre à leur sujet. C'est une image d'épuisement qui résume par exemple le souvenir que la vieille Madame Chenevert a laissé à son fils Alexandre. Se rappellant la joie que lui procurait, quand il était enfant, les visites de sa parenté, il revoit en même temps les contraintes qui étaient imposées à sa mère à cette occasion: “épuisée de corvées, la pauvre créature accueillait ce surcroît de besogne comme un soulagement à ses peines. "15 Dans cet exemple est mis en évidence le prix que coûte à la mère de famille le modeste plaisir de recevoir les siens. Et la bonne volonté de ce personnage devient rétrospectivement pathétique pour son fils qui se souvient d'elle comme pas bien forte "mais si vaillante" 16 .

La vieille Cousine Martine qui a eu quatorze enfants et vient de la ville pour revoir les lieux de sa vie passée avant de mourir apparaît comme n'ayant jamais réalisé l'étendue de l'asservissement quotidien qui a été le sien. Devant le spectacle du fleuve qu'elle retrouve, son visage n'exprime que la joie. Mais, en se déchaussant pour marcher dans l'eau, elle révèle « des pieds d'enfants, mais malmenés à ne pas le croire $\$ 17$. La narratrice comprend alors que c'est sur ces pauvres pieds que Martine a traversé sa vie difficile. Et celle-ci se rappelle soudain qu'en effet, ils l'ont tenue debout parfois "le temps de repasser d'affilée vingt-cinq chemises d'hommes $\$ 18$. II semble que, proche de mourir, cette mère fictive prenne conscience pour la première fois du sacrifice qu'elle a fait de sa personne.

Un autre exemple de l'humble sacrifice fait par la mère de famille est celui d'une émigrée polonaise fixée depuis sa jeunesse au Manitoba. Dans Un jardin au bout du monde, une nouvelle qui donne son titre au recueil montre Loubka évoquant son existence à travers tout ce qu'elle a fait de ses mains: “des oreillers également gonflés de plumes d'oies, des courtepointes colorées, sans compter les milliers de repas qu'elle avait préparés, et toutes ces basses besognes ingrates, comme de tuer de la volaille ou de recueillir le sang chaud du cochon "19. Habituée qu'elle est à s'effacer devant autrui, sa vie ne lui paraît mériter aucun respect et la peur la saisit "à la seule idée du dérangement à cause d'elle ${ }^{20}$. Sans nouvelles de ses enfants, Loubka vit seule avec son mari qui, depuis des années ne lui adresse plus la parole. Se voyant proche de la mort, elle s'interroge sur sa vie. Mais devant le vide d'une existence dans laquelle rien ne l'a jamais concernée, cette question reste sans réponse.

II arrive que l'accablement soit montré comme générant, chez les mères, une sorte de frigidité affective qui est exprimée de manières diverses. C'est le cas dans une nouvelle de La Route d'Altamont où la jeune Christine observe le déménagement de Mrs. Smith qu'elle voit comme une "pauvre créature lasse et couverte de poussière, „21 incapable de montrer le moindre sentiment à l'égard des lieux qu'elle quitte et du petit chien de la famille qu'elle abandonne. L'accablement physique est montré ici comme asséchant les ressources affectives du personnage. La servitude qu'a connue Madame Laplante dans Bonhour d'Occasion semble avoir eu la même conséquence. Elle a accompli plus que sa part de tâches domestiques: ayant eu quinze 
enfants, elle les a vêtus en filant, tissant et cousant, les a nourris et leur a enseigné leurs prières. Mais le sens du devoir avec lequel elle a assumé ces obligations semble avoir tari en elle toute source d'affection et ses enfants qu'elle n'a jamais embrassés, n'ont pu espérer d'elle «aucun aveu de tendresse "22. Devenue vieille, elle résume son existence en disant qu'elle a enduré son purgatoire sur terre. Et aux yeux de sa fille, elle apparaît comme «une négation obstinée de tout espoir»23. Cet état d'impuissance qui engendre un désespoir vague et envahissant transcende d'ailleurs les milieux et on le retrouve dans une nouvelle, La voix des étangs, où Éveline à qui sa fille annonce qu'elle veut devenir écrivain exprime, dans un geste désolé et impuissant, le méme pessimisme que Madame Laplante en laissant à sa fille ce message: "L'avenir est une chose terrible. C'est toujours un peu une défaite $\gg 24$.

Ainsi s'exprime la démission des femmes de cette œuvre, que la vie adulte et tout particulièrement le rôle maternel commandé par le conformisme du milieu, prive d'un épanouissement personnel. Pour elles, l'avenir est en effet cette débâcle de l'ego sacrifié aux exigences de la famille avant d'avoir même pu se découvrir. Sous une forme plus discrète, le message fataliste que la femme d'Alexandre Chenevert transmet à sa fille est le même: ‘ mieux vaut endurer, ma fille, prends ma parole»25.

II arrive que, pour certaines femmes, l'accablement soit réduit à un malaise moral. Dans ce cas, leur vie relativement aisée dissimule à leur entourage le sens du désespoir larvé qui les détruit. C'est le cas, par exemple, de la neurasthénie dont souffre Elizabeth Beaulieu dont on dit, dans La Rivière sans repos, qu'elle est « malade des nerfs " 26 . Sa servante esquimaude observe avec stupeur "l'incompréhensible peine de sa patronne»27. C'est après son troisième accouchement que la dépression de celle-ci empire. Isolée dans une existence qui ne lui convient pas, elle projette sur ses enfants sa sombre mélancolie se demandant "pourquoi on les met au monde, au fond ${ }^{28}$. On ne sait pas si ce qui désespère le plus ce personnage, c'est de vivre une existence taillée pour un autre ${ }^{29}$ ou d'être condamnée à un rôle maternel pour lequel elle n'a aucune vocation. Mais il ressort de sa condition qu'elle accrode à Elsa, sa servante, “ l'attention d'une prisonnière à une autre prisonnière dans la même cage»30.

Madame Létourneau, qui vit également dans l'aisance, présente aussi des traits évidents d'aliénation intérieure dans Bonheur d'Occasion. Croyant devoir servir de trait d'union entre son mari et son fils, elle est devenue comme un miroir qui reflète "avec excès la vivacité de son fils et la grave dignité de son mari 31 . Totalement effacée derrière eux, elle emprunte successivement l'identité de l'un et de l'autre et son discours exprime son incohérence intérieure.

Dans le même roman, toutes les servitudes des mères semblent s'additionner dans le personnage de Rose-Anna. Elle est montrée comme perdant pied progressivement dans la lutte quotidienne qu'elle mène contre la misère, les tâches harassantes et l'angoisse. Se comparant à son mari, Azarius, elle 
constate avec amertume qu'il est resté "jeune et beau de sa santé inaltérable»32 tandis qu'elle révèle "des marques si évidentes de fatigue et d'usure "33. Elle souffre de voir son corps, dont les grossesses successives lui enlèvent le contrôle, périodiquement aux mains d'autrui et d'avoir alors si terriblement besoin de secours. Et avant l'accouchement de son douzième enfant elle sombre dans une profonde dépression qui lui fait souhaiter mourir avec une profonde envie de repos. Par ailleurs, se souvenant des espoirs de sa jeunesse, elle discerne un contraste pitoyable entre la pauvreté de son foyer, le désordre qui l'entoure et la journée de son mariage «claire, limpide, avec des sons de cloche "34. Se sentant abusée par son destin, elle pense alors que c'est « une dérision ces pauvres jours de grâce au commencement de la vie»35. Quoique très attachée aux siens, elle découvre avec une stupeur douloureuse qu'elle perd progressivement le contact avec tous les membres de sa famille. Après avoir longtemps relevé tous les défis, Rose-Anna finit par s'abandonner à la fatigue qui l'a vieillie avant l'âge et cette démission s'incarne pour sa fille Florentine, dans un certain geste las et futile qui était jusque-là celui de sa grand-mère. Faisant le bilan de son existence, RoseAnna attribue l'accélération de son vieillissement, les souffrances de son corps et la plupart de ses humiliations à sa condition de femme. C'est ce qui lui fait repousser à chaque accouchement son désir profond d'avoir une fille afin de souhaiter "mettre au monde un enfant mâle qui souffrirait moins qu'elle »36.

Si le personnage de Rose-Anna résume à lui seul la condition maternelle dans l'œuvre, on trouve aussi dans Ces enfants de ma vie, une fresque de victimes qui offre un éventail complet de toutes les formes précédentes d'aliénation. La mère de Nikolaï, Anastasia, fabrique avec talent des fleurs de tissu pendant les mois d'hiver. Mais cette modeste activité personnelle est contrólée par son mari qui veille farouchement sur ses créations pour les vendre. Méme l'exercice de sa créativité ne parvient pas a lui restituer son autonomie. La mère du petit Clair, elle, n'a pas de loisir de profiter de sa liberté car, abandonnée par son mari, elle doit travailler sans relâche pour élever seule son jeune fils. Accablée de fatigue et d'inquiétudes, elle vit dans la crainte de ne pas suffire à sa tâche d'éducatrice. Présentées comme des victimes de l'autorité ou de l'absence du conjoint, ces mères ignorent toute forme d'autonomie. II arrive même que la maternité soit montrée comme mettant en danger la vie des femmes. C'est le cas de Madame Badiou, qui est victime d'accouchements interminables comme le savent ses voisins qui racontent qu'elle est restée "trois jours la dernière fois encore à hurler »37. Quant à la mère du petit André, elle est décrite comme passant six mois de ses grossesses allongée, prisonnière de "sa terrible tâche de pourvoyeuse de l'espèce "38. Son rôle est d'ailleurs si écrasant que son fils André doit l'aider à l'assumer. On voit briller "une joie maternelle "39 dans les yeux du petit écolier mais bientôt dépassé par cette tâche, il doit quitter l'école et ses "grands yeux tracassés 40 révèlent sa parenté avec les "prisonnières".

Le spectacle de leur destin sacrifié provoque dans l'entourage de ces personnages un malaise fait de compassion et de culpabilité. Et, qu'ils soient 
privés de vie personnelle par l'excès des tâches matérielles ou par le fait de ne participer en rien à leur destinée, qu'ils appartiennent à la classe paysanne et ouvrière ou à la petite bourgeoisie, tous sont montrés, à un titre ou à un autre, comme auréolés de la pitié qu'ils inspirent. Ce sentiment ambigu apparaît comme la réponse du milieu au spectacle des «prisonnières». La culpabilité qui le compose étouffe chez certains le goût de vivre tandis que chez d'autres, il nourrit une révolte qui, les poussant vers de périlleuses sublimations, hypotheque le plus souvent leur existence.

\section{Le va-et-vient de la pitié}

Pour évoquer toutes ces héroïnes, même quand elles ne se profilent que très secondairement dans les récits, l'auteur retrouve toujours le même champ sémantique centré autour de l'adjectif «pauvre»: "cette pauvre créature", "ses pauvres petits pieds", "cette pauvre vie", "la pauvre âme épuisée", "son pauvre sourire", "le pauvre regard", "sa pauvre âme», "ses pauvres mains", " pauvre maman, va! pauvre maman! ", “ la pauvre créature", "pauvre maman, pas bien forte "41. On voit ainsi successivement la personne et l'existence de ces mères traitées avec apitoiement. Certaines parties de leur corps qui participent de leurs tâches - les mains usées ou les pieds fatigués - bénéficient à l'occasion de la pitié d'ensemble. Et leur visage enfin vient confirmer par le regard et le sourire leur accablement moral et leur impuissance.

Quelquefois, d'autres épithètes viennent préciser le sens de celui qui est privilégié: " doux visage d'expression timide", "les yeux immenses tristes et doux", "doux visage blond de poupée", "timide, aimante et faible»42. II ressort de ces exemples que l'impuissance de ces personnages s'accompagne de faiblesse et que leur condition est intériorisée dans une sorte de sublimation morale. En effet, au lieu de générer en elles la révolte, leur état n'entraîne qu'un effacement atimide" et une "tristesse" qui se métamorphosent, à l'occasion, en une douceur qui signale leur démission. A l'image de leur écrasement se substitue donc celle de leur résignation morale pour faire de ces personnages d'obsédants martyrs.

La pitié générée par l'image de la mère peut apparaître parfois comme contagieuse dans cet univers. On la voit par exemple contaminer toutes les relations des membres de la famille Chenevert. Entre eux, ce sentiment ambigu a fini par remplacer tous les autres. II n'est probablement pas étranger, d'ailleurs, sous la forme d'un apitoiement chronique envers soimême, au malaise dont souffre le héros. A la compassion qu'il a ressentie pour sa mère a répondu chez celle-ci une pitié machinale et permanente envers lui. De son vivant, elle l'abreuve d'exclamations qui se résument dans son expression favorite: "pauvre, pauvre toi!"43 Dans son dépit de ne pouvoir lui transmettre son expérience, elle a ainsi fait planer sur lui la menace d'un toujours hypothétique cataclysme. Devenu vieux, lui-même s'entend avec stupeur répéter la même épithète en parlant à son camarade de bureau dont il ne parvient jamais à capter l'attention: "pauvre, pauvre Godias »44. 
Entre les membres de la famille Chenevert, on assiste à une sorte de surenchère de la pitié. C'est celle, impuissante, que ressent Eugénie pour son mari. A sa fille qui lui fait des confidences, elle conseille de parler bas pour ne pas réveiller son père: "le pauvre homme! il dort »45. Un jour qu'Alexandre a fait un gros effort d'imagination pour apporter à Eugénie hospitalisée un bouquet, celle-ci manifeste une stupeur apitoyée: «Pauvre vieux! Des fleurs! »46

Alexandre rend bien à sa femme la pitié qu'elle lui manifeste et il en confond d'ailleurs volontiers les causes avec une malédiction liée à son sexe. Réfléchissant au fait que les hommes et les femmes sont irrémédiablement séparés sur terre par leurs misères respectives, il croit deviner qu'à tout prendre, "celles des femmes sont peut-être les plus dures »47. Observant sa fille Irène qui s'est mariée contre son gré et tente de lui dissimuler ses ennuis conjugaux, il ressent une pitié qui devient « une amertume débordante »48. Par ailleurs, reconnaissant chez elle une angoisse qu'il lui a léguée, il la plaint: "Pauvre petite fille». Sa pitié englobe aussi le fils d'Irène: "Pauvre petit Paul», car leurs liens familiaux lui apparaissent comme une "chaîne de transmission de la douleur \$49. Sa commisération rencontre d'ailleurs celle de sa fille dans un passage très révélateur. N'étant pas parvenu à établir de vrai dialogue avec elle au cours d'un séjour qu'elle a fait chez lui, une émotion envahit Alexandre au moment où s'ébranle l'autobus qui emmène sa fille et tous deux tentent de communiquer dans le bruit du démarrage: "pauvre, pauvre papa, tâche de te faire soigner", crie Irène tandis que son père lui jette un conseil apitoyé : “Attention... ta petite santé »50. Entre eux, l'apitoiement semble être devenu le seul langage affectif.

La pitié qui a d'abord été inspirée à Alexandre par l'image de la servitude maternelle a éveillé en lui une culpabilité vague et envahissante qui a corrompu ses rapports affectifs comme le montre dans un raccourci révélateur l'irritation que lui cause sa femme un jour où le chagrin qu'il lui a fait la lui rend "soudainement insupportable»51. Cette pitié qui s'étend progressivement à tous les membres de la famille devient pour eux un alibi. En se traitant avec apitoiement, ils atténuent la culpabilité que leur inspire leur froideur mutuelle. Transformant cette dernière en sensiblerie, la pitié donne en effet l'illusion de colmater les brèches creusées par l'indifférence dans l'unité familiale. L'origine de ce silence affectif dans lequel s'épanouit ainsi ce sentiment complexe, on la trouve dans la compassion navrée et dévorante que l'image de la condition maternelle a inspirée à Alexandre. Avec le défaitisme qu'elle lui a communiqué, sa mère lui a ôté la force de vivre avant même qu'il n'ait commencé sa vie adulte 52 .

\section{Les sublimations}

Si le spectacle de l'asservissement maternel génère chez tous ses témoins un malaise apitoyé, la narratrice nous montre que certains d'entre eux en sont marqués plus profondément. Nous avons vu que cette expérience 
a épuisé chez Alexandre Chenevert des forces vives, ce qui le condamne à une existence dont il ne se sent jamais solidaire. Chez Florentine, la fille de Rose-Anna, dans Bonheur d'Occasion, il a un effet différent. L'observation quotidienne de la condition maternelle a généré en elle une honte irritée, assortie de culpabilité. La révolte que lui inspire l'existence de Rose-Anna trouble l'élaboration de son identité féminine. En effet, elle entretient avec sa mère des rapports ambigus. D'une part, sensible, elle sait discerner "la souffrance, le courage écrits sur ce visage $\$ 53$. Mais d'autre part, elle est saisie d'horreur devant le spectacle de la condition féminine que lui offre sa mère et elle refuse de s'identifier à cette image de misère et de démission. La narratrice nous montre Florentine faisant l'expérience de la compassion impuissante. Un jour qu'elle a donné de l'argent à sa mère dans un mouvement spontané, elle découvre l'inutilité de ce geste puisqu'il a fait naître en Rose-Anna tant de désirs qu'elle en est plus pauvre après qu'avant. Et, d'un coup, toute "la joie " 54 que Florentine avait éprouvée se change "en fiel»42. Au "ravissement infini»42 qu'elle a d'abord ressenti à l'idée de soulager un peu Rose-Anna de sa misère, succède la "stupeur douloureuse "42 de ne rien pouvoir pour elle. Mais Florentine qui est représentée comme un être de volonté refuse de se laisser écraser par cette expérience et pour se protéger devant l'affligeant spectacle de sa mère, il est précisé que son cœur se durcit pour se mettre " en garde contre l'attendrissement „55. De la joie au fiel, de l'élan au raidissement, de la tendresse à la froideur, Florentine est montrée comme faisant l'expérience de la pitié impuissante. Volontaire et indépendante, elle tente cependant de se défendre, car une violente révolte naît en elle à l'idée de connaître le même asservissement que Rose-Anna et la vie de celle-ci lui apparaît comme "un long voyage gris, terne, que jamais, elle, Florentine $\$ 56$ n'accomplira.

\section{La sublimation sociale}

Cette revendication passionnée prend la forme d'une sublimation sociale. Croyant trouver en Jean Lévesque les éléments d'une existence supérieure à celle de sa famille, elle tente, dans un défi à son destin, de le séduire et se laisse plus ou moins violer par lui. Abandonnée, elle se découvre enceinte et voit «le piège $" 57$ qui lui a été tendu. Alors, se retrouvant, comme Rose-Anna qui subit une douzième grossesse, réduite dans son corps, elle éprouve "plus fort encore que sa peur, un indicible mépris pour sa condition de femme»58. Et dans l'affrontement qui les oppose quand Rose-Anna découvre le secret de la fille, il n'y a place pour aucune solidarité. Au contraire, une haine mutuelle naît entre elles de l'aversion que leur inspire leur condition de femmes. La mère regarde sa fille «sans pitié, sans amitié, sans bonté: rien que de l'horreur plein les yeux»59. Et à cet appel "d'être traqué » 60 que lui lance Florentine, elle ne répond que par le silence d' une chose inerte, indifférente, à demi-enfoncée dans le sommeil „61. Quant à Florentine, elle n'oublie pas le silence réprobateur qui a répondu à sa détresse et, plus tard, elle part se marier, en traitant Rose-Anna comme une étrangère. Elle plonge ainsi sa mère dans un désespoir qui fait souhaiter à 
celle-ci " se cacher quelque part, cacher son visage, rester longtemps seule, toute seule»62. Entre elles, on le voit, la honte de leur sexe rend toute communication véritable impossible. La mère offre à sa fille une intolérable image d'échec et de démission. Et celle-ci lui impose en retour une déchirante désapprobation.

Mais la fille de Rose-Anna tente de reprendre en main son destin. Sa courte expérience lui a appris que la vie est un rapport de forces et que "l'argent c'est ce qui compte dans Saint-Henri comme ailleurs "63. Pour dominer son destin, il lui faut abuser de son meilleur ami, Emmanuel, mais en l'épousant elle se libère d'un certain nombre de servitudes qui, nées de la misère, ont été celles de sa mère. En légitimant sa grossesse, elle fait, «par la force seule de sa volonté "64 d'une situation qui avait failli la marginaliser définitivement, un tremplin qui l'élève au niveau des Létourneau, les anciens employeurs de sa mère. L'auteur ne nous dit pas si elle y trouve le bonheur. Dans ces moments décisifs de la lutte pour l'existence. Florentine avance aveuglément vers ce qui lui apparaît comme essentiel. Et il semble que, dans les remous de sa révolte, elle sacrifie, aux exigences de l'indépendance et de l'orgueil, comme aux menus plaisirs de la vanité, une forme de son intimité. Mais aux antipodes du destin de sa mère fait d'illusions, d'amour spontané et de don de soi, Florentine semble trouver dans le calcul et la sécurité petitbourgeois une identité sociale et une indépendance qui lui tiennent lieu de havre.

\section{La sublimation raciale}

Toutes les filles révoltées contre l'image maternelle n'ont pas le même succès que Florentine dans le combat pour l'existence. Elsa l'Esquimaude qui vit à Fort-Chimo dans La Rivière sans repos jette à son destin un défi plus périlleux. Elle blâme sa mère Winnie de ne jamais chercher à s'élever. Quand elle l'observe, elle voit en elle l'être auquel elle tient le moins à ressembler, "peut-être même sa pire ennemie "65.

Le défi d'Elsa se confond d'abord avec la conquête du progrès qu'elle identifie bientôt avec l'enfant blanc qu'elle a eu d'un G.I. et devient une sorte de sublimation raciale: "A son premier regard sur cet enfant aux yeux clairs, aux cheveux pâles, venu d'elle, la noiraude, elle s'était sentie sombrer dans un abîme d'amour ${ }^{66}$. Elle est montrée cherchant pour l'amour de son fils à s'intégrer à un autre monde que le sien et devenant comme les jeunes femmes blanches qui se proposent toujours "des buts de plus en plus difficiles à comprendre 36 . Elle travaille par exemple comme une forcenée pour assurer à son fils les vêtements et les jouets qui sont ceux des enfants de sa patronne. Bientôt, la fatigue et l'usure commencent à la marquer. Pourtant, son visage "crispé par tant de fierté "68 s'oppose d'abord au visage a affaissé et terni 69 de sa mère et semble révéler une lutte victorieuse. Mais l'enfant grandissant conçoit une honte de ses origines et de sa mère aussi puissante que celle qu'avait inspirée Winnie à Elsa. Et la jeune femme voit progressivement sa vie perdre son sens. Au moment où un prétendant de sa race lui 
offre une intégration, elle laisse son fils contrarier ce début d'idylle, faisant passer sa passion maternelle avant son avenir personnel.

Enfin, son fils ayant quitté Fort-Chimo, elle voit sa vie se défaire et glisse dans "la fainéantise et l'habitude de toujours rêvasser" 70 qu'elle reprochait tant à sa mère. Vieillie avant l'âge par la fatigue et le chagrin, elle ressemble tant à cette dernière que ses voisines la prennent pour Winnie revenue sur terre. Après avoir passionnément voulu dépasser sa mère, Elsa se retrouve un peu plus harassée qu'elle, prisonnière du même destin honni auquel, impuissante, elle finit par s'abandonner.

On voit que chez Elsa comme chez Florentine, l'image de la détresse maternelle engendre une révolte. Indépendantes et passionnées, elles croient pouvoir, elles, choisir une meilleure part. Florentine dont la grossesse scandaliserait le milieu ouvrier de Saint-Henri la légalise par un mariage de tête qui assure son avenir matériel. Les images de mort des dernières lignes de Bonheur d'Occasion nous sont confirmées par l'auteur qui, dans Fragiles Lumières de la Ville, nous apprend qu'Emmanuel ne revient pas de la guerre. Florentine devient donc veuve. Mais protégée par les défenses de l'orgueil et de l'intérêt, elle s'est assurée la complicité protectrice de la société.

II en va tout autrement pour Elsa qui, violée par un G.I. qu'elle ne dénonce pas, se retrouve enceinte, elle aussi. Dans son milieu, elle peut élever sans honte le bébé rose et blond qu'elle idolâtre. Elle entreprend alors un combat inégal contre la société en croyant pouvoir modeler de ses mains nues une existence de petits bourgeois pour son fils. Étrangère chez les Blancs, elle ne parvient pas à se réinsérer parmi les siens. Finalement, le fantôme de sa mère lui apparaît comme pour réveiller en elle l'atavisme de sa race. II réveille en elle des tendances profondes à la démission et au fatalisme, qu'elle n'a surmontées jusque-là que par "un perpétuel élan d'amour ${ }^{71}$. On voit que le courage, la fierté et l'amour ne suffisent pas à Elsa pour relever avec succès le défi qu'elle s'est lancé. Après s'être révoltée contre l'image maternelle, elle se montre incapable de se choisir. Quoique refusant ce qui lui apparaît comme la démission de Winnie, son premier acte libre consiste à se mettre au service de son enfant blanc dont elle se fait une sorte de dieu. Investissant toute son existence dans cette passion, elle perd sa raison de vivre avec le départ de son fils. $A$ la défense de sa personne, Elsa préfère un fallacieux idéal, chez elle, la révolte contre l'image maternelle est montrée comme plus suicidaire que libératrice.

\section{La sublimation mystique}

Toutes les filles fictives de l'œuvre n'offrent pas le spectacle d'une révolte ouverte. II arrive que leur conduite suicidaire prenne une forme plus feutrée. C'est le cas, par exemple, de la sœur de Florentine, Yvonne. Cette fillette silencieuse ne se signale aux siens que par une "piété excessive "54. On voit cependant que son chagrin devant les souffrances quotidiennes de sa mère a déjà entamé sa confiance en l'avenir. Elle est montrèe comme 
observant Rose-Anna avec "une expression de tendre pitié, de protection même, plutôt que de muet reproche comme autrefois "72. Une tendre pitié protectrice a pris la suite de sa timide révolte et, un jour où sa mère lui demande si elle pense se marier plus tard, elle répond sans hésitation qu'elle veut devenir religieuse. On apprend par ailleurs qu'elle a offert sa vie à Dieu pour la guérison de son frère Daniel. Contrepoint de la révolte, la résignation sublimée prend chez elle la forme du renoncement mystique, défi et sacrifice permettant également de prendre ses distances par rapport à l'accablante image maternelle. Rose-Anna ne s'y trompe pas et comprend qu'entre elles, "une distance infranchissable ${ }^{73}$ vient de s'établir.

II semble donc que la révolte de la jeune génération féminine de cette cuvre tende toujours violemment vers l'affirmation même désespérée, de sa différence. Optant comme Florentine pour l'argent, les jeunes filles visent avant tout à échapper à l'image obsédante du navrant destin maternel.

\section{La sublimation littéraire}

II arrive, exceptionnellement, que le sacrifice soit montré comme n'aliénant pas, en apparence, celles qui l'assument. C'est qu'alors, la narratrice opte pour un ton valorisant qui place ses héroïnes dans le monde merveilleux qui est celui de l'idéal. C'est le cas, dans La Petite Poule d'Eau, de Luzina ${ }^{74}$. Opposé à l'univers de misère et d'échec de La Riviere sans repos et de Bonheur d'Occasion, le monde de Luzina semble incarner les valeurs bibliques de paix et d'amour. Elle-même apparaît comme guidant l'épanouissement des siens et de ses proches en les aidant à s'apercevoir qu'ils ont "des raisons d'être heureux"75.

Malgré l'isolement dans lequel elle vit dans l'île de la Petite Poule d'Eau, elle réussit à reprendre partiellement le contrôle de son existence en métamorphosant, grâce à son imagination créatrice, ses devoirs maternels en actes personnels. On assiste chez Luzina à une sublimation littéraire qui lui fait ainsi transposer son existence quotidienne et romancer sa vie. En ce sens, on peut rapprocher cette idéalisation de celle d'Éveline à la fin de la nouvelle Les Déserteuses. On la voit, par exemple, transformer en «unique aventure " 76 le voyage difficultueux que ses accouchements quasi annuels lui imposent pour se rendre à Sainte-Rose-du-Lac. Elle fait un exploit de ce déplacement périodique dans lequel, à certaines saisons, elle risque sa vie. Et elle est montrée comme tirant de multiples joies des lieux qu'elle traverse à cette occasion et des gens qu'elle rencontre. N'est-elle pas restée en correspondance pendant dix ans avec une amie qu'elle s'est faite à cette occasion? De ce voyage qui est chaque fois pour elle une nouvelle découverte du monde, elle rapporte de quoi alimenter les récits qu'elle fait à sa famille pendant des mois, regénérant ainsi aux yeux des siens l'image maternelle.

Elle parvient par ailleurs à obtenir du gouvernement une école pour ses enfants. Grâce à elle, ils peuvent bénéficier d'une instruction qu'elle n'a pas reçue. Mais après toutes les conquêtes qu'elle a poursuivies gaiement pour 
les siens, Luzina fait avec leur départ l'expérience du découragement et même d'une certaine honte de soi que son militantisme familial avait comme exorcisés jusque-là. Privée de ses "voyages", elle perd le contact avec l'extérieur qui nourrissait son enthousiasme. De plus, ses enfants qui vont à l'Université la rendent plus consciente de son ignorance. Elle les sent très supérieurs à elle et quand elle leur écrit, pour ne pas leur faire honte, elle demande à sa cadette de tracer les adresses sur ses enveloppes. II ne lui reste donc que l'amère fierté d'avoir promu les siens à une existence libre qu'elle n'a pas connue. A la honte que lui inspire son infériorité répond chez ses enfants une pitié culpabilisante: "les enfants instruits de Luzina avaient un instant le cœur serré, comme si leur enfance là-bas, dans liîle de la Petite Poule d'Eau, leur eût reproché leur élévation "77. Plus décisive que la distance qui les éloigne de leur mère, du Québec au Manitoba, entre Luzina et ses enfants est née la différence que créent l'instruction et l'émancipation.

Le paradis de la Petite Poule d'Eau laisse donc place à l'échec et celui-ci vient nourrir l'interrogation que pose l'œuvre sur le sens de la liberté pour les personnages féminins. En mettant la sienne au service de ses enfants, Luzina a connu de multiples joies. En sublimant son existence, elle a effacé ses servitudes. Mais, ses enfants partis, le réel reprend le dessus sur l'idéalisation. Elle découvre, en particulier, que le privilège de s'instruire qu'elle leur a donné est justement ce qui finalement la sépare d'eux. Après avoir été le levain de l'existence des siens, Luzina est montrée vieillie, les mains vides. On découvre alors que la sublimation qu'elle a pratiquée jusque-là n'était nourrie que par son amour maternel et que, pour son seul bien, elle n'est pas capable de la poursuivre. L'impuissance qui succède au pouvoir qu'elle a exercé sur le bien-être de sa famille lui inspire honte et mélancolie. En somme, après avoir été longtemps valorisée par le rôle maternel, elle se retrouve aussi isolée que Rose-Anna qui a été écrasée par lui. Pour décrire ce qui les sépare de la génération qui les suit, la narratrice utilise des expressions équivalentes en qualifiant cet éloignement de "distance définitive" et de "distance infranchissable". Rose-Anna qui maudit sa condition de femme et Luzina qui semble l'avoir glorifiée sont, à la fin de leur vie, égales dans l'effacement de leur personne devant le rôle maternel. Les misères de l'une et l'existence sublimée de l'autre conduisent également à leur solitude finale. Toutes deux, comme d'ailleurs Elsa de La Rivière sans repos, connaissent la honte de se sentir inférieures à leurs enfants.

Si les sublimations que nous avons observées se soldent par des échecs divers, souvent tragiques, une idéalisation apparaît dans l'œuvre, qui semble apporter au personnage féminin à la fois un certain bonheur d'aimer et l'indépendance.

\section{La sublimation professionnelle ou le recours mythique}

Dans le prolongement de la métamorphose du personnage d'Éveline telle que nous l'avons vue à la fin de Les Déserteuses dans Rue Deschambault, et dans la perspective de l'existence idéalisée de Luzina, on trouve dans 
l'œuvre un personnage féminin dont l'existence entière se déroule dans un climat surnaturel. II apparait déjà sous les traits de Christine à la fin de Rue Deschambault et devient l'héroïne de Ces enfants de ma vie. Il s'agit de l'institutrice. Ce personnage féminin qui ne connaît pas les servitudes des mères de l'œuvre exerce cependant une maternité symbolique auprès de ses écoliers. Dévouée à la communauté qu'elle sert, elle ne perd pas pour autant sa liberté de mouvement et se tient à la croisée de " ces carrefours et chemins passant que sont les livres $\$ 78$. Maitresse de son existence, elle peut la mettre momentanément au service des enfants qui lui sont confiés, car le dévouement qu'elle leur offre ne compromet pas son autonomie.

La ferveur avec laquelle ce personnage féminin est décrit est aussi passionnée que la compassion réaliste qui caractérisait les portraits de mères fictives de l'œuvre. Et on ne peut s'empêcher de voir dans cette femme libre et heureuse le contrepoint parfait de la mère écrasée et asservie. En effet, dans l'espace privilégié de sa classe, la maîtresse d'école a les pleins pouvoirs et, ses écoliers à ses pieds tels "Vendredi à ceux de son maître»79, elle est montrée comme radicalement opposée aux mères affligées de ses écoliers. $A$ l'égard de celles-ci, elle ne se désolidarise pas et elle pleure avec elles «sur la misère féminine ${ }^{80}$. Grâce à la distance que sa liberté et son pouvoir établissent entre elle et les enfants qui lui sont confiés, toute la gamme de l'amour maternel idéalisé trouve à s'épanouir. Et dans ce commerce affectif privilégié, il est dit que la jeune fille trouve satisfaction car elle provoque l'amour qu'elle est le plus apte à goûter en étant " adorée à distance comme une bonne étoile qui guide à travers la vie „81. Très loin de l'asservissement des mères, ce personnage concilie l'indépendance et le don de soi. L'institutrice apparaît comme la médiatrice victorieuse entre les mères écrasées par leur rôle et les filles que leur révolte marginalise. Dans la conformité d'un rôle privilégié qui protège son autonomie et lui donne un pouvoir, dans une maternité sublimée qui n'exclut pas la tendresse, ce personnage féminin fait l'expérience d'une plénitude. C'est celle que Christine confie dans Rue Deschambault : " Je ne le savais pas tout à fait encore - nos joies mettent du temps à nous rattraper - mais j'éprouvais un des bonheurs les plus rares de ma vie»82. L'obsédante présence des mères dans l'œuvre révèle en effet l'importance de la maternité dans cet univers imaginaire. Or le rôle de l'institutrice parvient à combler cette exigence comme celle, aussi puissante, de l'autonomie. C'est de cette double satisfaction que naît, semble-t-il, le bonheur exalté de la maîtresse d'école.

D'un point de vue idéologique, le tableau de la condition des femmes offert par l'œuvre de Gabrielle Roy peut paraître assez frustrant. En effet, par la dénonciation généreuse et attentive qu'elle fait du sacrifice des femmes, en particulier dans leur rôle de mère, la narratrice s'élève contre une certaine réalité et sert les thèses féministes. Mais la récupération mythique du personnage de l'institutrice dans l'image d'un pouvoir individuel lié au talent personnel, et indirectement à l'art, efface toute solidarité à l'égard des autres personnages féminins. L'institutrice seule est sauvée et oubliées toutes les femmes asservies qui défilent dans l'œuvre. II semble ainsi que la narratrice 
n'assume pas complètement la compassion qu'elle montre à l'égard des créatures les plus défavorisées. En faisant de la maîtresse d'école un personnage mythique, de son bonheur un exploit, elle rend miraculeuse la seule libération féminine de l'œeuvre. Et ce statut d'exception condamne indirectement à l'impuissance toute la condition féminine qui ne relève pas de Iui.

D'un point de vue littéraire, ce passage de la personne asservie par le groupe à l'individu triomphant conduit au coeur de cet univers imaginaire. Car l'institutrice qui est la médiatrice entre les livres et l'enfance est aussi à la croisée de la connaissance et de la tendresse, de la liberté et de l'attachement. Elle résoud donc la contradiction vécue par les femmes aliénées de l'œuvre. Nous ne confondrons pas la narratrice avec l'auteur, sa vie avec son œuvre, car il est clair que, pour être utilisable, il faudrait que la biographie de l'écrivain «soit donnée comme un des traits de l'œuvre elle-même "83. Mais sans rien expliquer, le rapprochement d'une déclaration personnelle faite par l'auteur éclaire la thématique que nous analysons. Notons en effet qu'elle confie avoir vécu cette dissociation intérieure que subissent ses personnages féminins: "Je cherche encore à concilier le besoin de liberté dont nous ne pouvons nous passer avec l'affection qui attache, la tendresse qui retient $" 84$. Elle précise aussi dans fragiles lumières de la terre: "de nos deux vies, la collective avec ses chaînes de responsabilités et la privée avec ses aspirations à l'indépendance, l'une sans l'autre est frustrée „05. L'image idéale de cette héroïne dont l'unité est un privilège, déclenche dans l'œuvre un lyrisme qui révèle manifestement la séduction qu'elle exerce sur la narratrice. Littérairement, elle est productrice de texte. A la gloire de l'individualisme et exaltant "l'idéal, la vocation, la réalisation de la personnalité» 86 , ce personnage mythique incarne dans cet univers imaginaire une perfection. II synthétise peut-être, par ailleurs, la somme des aspirations d'une vie. Mais sa présence exceptionnelle au milieu du tableau, réaliste par ailleurs, de la condition féminine confirme que, dans cette œuvre, le seul rôle féminin apportant une plénitude relève du merveilleux.

Dans sa description de l'asservissement des femmes, Gabrielle Roy exprime une indignation contre l'injustice de la condition féminine. Le personnage mythique correspond, lui, à l'optimisme de la narratrice et à sa foi dans le pouvoir de l'art et de la tendresse. A la division vécue par tous les personnages féminins de l'œuvre ${ }^{87}$, il oppose une triomphante unité réalisée grâce à un talent personnel. En ce sens, le point de vue de Gabrielle Roy sur la condition féminine est aristocratique. Un seul personnage est sauvé et il le doit à son imagination créatrice. La vision qu'a la narratrice de l'artiste ressemble à celle des écrivains romantiques. Comme eux, elle croit à l'aristocratie des artistes et à l'art comme panacée. Dans cette perspective, le sort des personnages féminins - dont mieux que personne Gabrielle Roy sait illustrer l'asservissement - repose sur une sorte de prédestination. Dans cet univers imaginaire, en effet, le talent, inné autant qu'imprévisible, reste pour les femmes la clé de toute libération. 
1. Dans cette œuvre, comme l'indique François Ricard (Gabrielle Roy, Fides 1975) * l'image de la mère est particulièrement riche et éclaire peut-être mieux que toute autre les implications contradictoires du geste créateur" (p. 32).

2. François Ricard, op. cit., p. 74.

3. Marc Gagné, Visages de Gabrielle Roy, Beauchemin 1973 (dans le chapitre “images et Espace” p. 97 à 133).

4. Gabrielle Roy, Rue Deschambault, Beauchemin, 1976, p. 99.

5. Ibid., p. 108.

6. Ibid., p. 138.

7. Ibid., p. 101

8. Ibid., p. 101.

9. Ibid., p. 110.

10. Ibid., p. 109.

11. Ibid., p. 113.

12. Ibid., p. 124.

13. Ibid., p. 130. L'amitié tient lieu à plusieurs mères de l'œuvre d'ouverture sur le monde. C'est le cas, comme pour Éveline, et de Luzina dans La Petite Poulo d'Eau et de Loubka dans Un jardin au bout du monde. L'une et l'autre entretiennent avec une amie lointaine une correspondance qui les tire de leur isolement.

14. Ibid., p. 136.

15. Gabrielle Roy. Alexandre Chenevert. Beauchemin 1973, p. 144.

16. Ibid., p. 155.

17. Gabrielle Roy. Cet été qui chantait. Les Éditions Françaises, 1972, p. 157.

18. Ibid., p. 157.

19. Gabrielle Roy. Un jardin au bout du monde. Beauchemin, 1975, p. 166.

20. Ibid., p. 168.

21. Gabrielle Roy. La Route d'Altamont. H.M.H., 1969, p. 174.

22. Gabrielle Roy. Bonheur d'Occasion. Beauchemin, 1967, p. 173.

23. Ibid., p. 172.

24. Gabrielle Roy. Rue Deschambault, op. cit., p. 247.

25. Gabrielle Roy. Alexandre Chenevert, op. cit., p. 146.

26. Gabrielle Roy. La Rivière sans repos. Beauchemin, 1971, p. 151.

27. Ibid., p. 191.

28. Ibid., p. 150.

29. Nous apprenons en effet que son mari préfère à celle du Sud la vie de l'Arctique, parce que, montrant à nu le dur visage de la vie, elle lui paraît finalement moins inhumaine. II envisage d'ailleurs de quitter un jour FortChimo parce que sa femme se n'y adapte pas, croyant que cet exil est la seule cause de ses dépressions.

30. Ibid., p. 152.

31. Gabrielle Roy. Bonhour d'Occasion. op. cit., p. 114.

32. Ibid., p. 79

33. Ibid., p. 79 .

34. Ibid., p. 311.

35. Ibid., p. 311 .

36. Ibid., p. 323.

37. Ces enfants de ma vie, Stanké, 1977, p. 119.

38. Ibid., p. 121.

39. Ibid, p. 112.

40. Ibid., p. 113.

41. Dans l'ordre: La Route d'Altamont, op. cit., p. 174 ; Cet été qui chantait, op. cit., 
p. 157; La Rivière sans repos, op. cit., p. 197 ; p. 313 ; Bonheur d'Occasion. op. cit., 104, 108, 114, 313, 315; Alexandre Chenevert, op. cit., p. 144 ; 155.

42. Ces enfants de ma vie, op. cit., p. 26; p. 117; Bonheur d'Occasion, op. cit., p. $113 ;$ p. $114 ; 377$.

43. Gabrielle Roy, Alexandre Chenevert, op. cit., p. 65.

44. Ibid., p. 65.

45. Ibid., p. 147.

46. Ibid., p. 131.

47. Ibid., p. 127.

48. Ibid., p. 140.

49. Ibid., p. 150.

50. Ibid., p. 150.

51. Ibid., p. 117.

52. Comme l'indique Gérard Bessette, le personnage d'Alexandre Chenevert et son destin ne peuvent se comprendre qu'à partir de ce lien de pitié-culpabilité qui l'attache à jamais à Élise Chenevert sa mère. (Trois romanciers québécois, Éditions du Jour, p. 204 et 208).

53. Gabrielle Roy, Bonheur d'Occasion, op. cit., p. 104.

54. Ibid., p. 109.

55. Ibid., p. 109.

56. Ibid., p. 104.

57. Ibid., p. 223.

58. Ibid., p. 223.

59. Ibid., p. 232.

60. Ibid., p. 232.

61. Ibid., p. 232.

62. Ibid., p. 314

63. Gabrielle Roy, Fragiles lumières de la Terre. Quinze, 1978, p. 173.

64. Ibid., p. 302.

65. Gabrielle Roy, La Rivière sans repos, op. cit., p. 162.

66. Ibid., p. 138.

67. Ibid., p. 165.

68. Ibid., p. 173.

69. Ibid., p. 197.

70. Ibid., p. 292.

71. Ibid., p. 292.

72. Ibid., p. 315.

73. Ibid., p. 317.

74. Jacques Allard a bien montré combien, dans ce roman, même le paysage, mis en relation avec ses habitants, "acquiert une dimension exceptionnelle, un caractère mythique». (Le Chemin qui mene à La Petite Poule d'Eau, Cahiers Sainte-Marie, vol. I. 1966, p. 58).

75. Gabrielle Roy, La Petite Poule d'Eau, Beauchemin, 1970, p. 31.

76. Ibid., p. 15.

77. Ibid., p. 165.

78. Gabrielle Roy, La Rivière sans repos, op. cit., p. 216.

79. Gabrielle Roy, Ces enfants de ma vie, op. cit., p. 15.

80. Ibid., p. 119.

81. Ibid., p. 210.

82. Gabrielle Roy, Rue Deschambault, op. cit, p. 293.

83. Tzvetan Todorov, Introduction à la littérature fantastique, Paris, Seuil 1970, p. 159.

84. Gabrielle Roy, Le Devoir, 18 mai 1974. 
85. Gabrielle Roy, Fragiles lumières de la terre, op. cit., p. 231.

86. Gabrielle Roy, La Petite Poule d'Eau, op. cit., p. 163.

87. Cette division dont Albert Legrand a montré que, marquant tout l'univers imaginaire de Gabrielle Roy, elle caractérise non seulement les personnages, mais aussi l'espace et le mouvement dans l'œuvre. (Gabrielle Roy ou l'être partagé, in Etudes Françaises, juin 1965). 